\title{
DESIGNING AND BUILDING A COST-EFFICIENT SURVEY DRONE
}

\author{
H. Kauhanen ${ }^{1}$, P. Rönnholm ${ }^{1}$, M. Vaaja ${ }^{1}$, H. Hyyppä ${ }^{1}$ \\ ${ }^{1}$ Department of Built Environment, Aalto University, P.O. BOX 14100, 00076 AALTO, Finland - (heikki.kauhanen, petri.ronnholm, \\ matti.t.vaaja, hannu.hyyppa)@aalto.fi
}

Commission I, ICWG II

KEY WORDS: Drone, camera, gimbal, design, simulation, assembly

\begin{abstract}
:
In this paper, we present a workflow how to design and implement a low-cost survey drone that meets the quality requirements of a much higher cost drone system. The technical specifications of available components and our design boundaries were applied in eCalc $\mathrm{RC}$ - xcopterCalc calculator in which the optimal setup was found by simulation. The main boundaries of design were derived from safety, operation time and payload capacity. Pixhawk 2 FCU, which is based on ArduPilot open source platform, was selected to handle autopilot and control functionalities. In addition, the system included a camera and a gimbal. The camera was controlled by FCU, which allows to geotag images using the on-board GPS data. The assembled survey drone was tested in a real survey mission. We successfully managed to complete a 13 minutes survey mission in mild wind conditions. According to simulation, the expected flight time range was between 9 and 15 minutes. In addition, simulation provided useful information on how the drone works under certain conditions such as working in extreme temperatures or high elevation locations as well as under heavy payloads. Even though our example was a survey drone, it is possible to use the same principles to design and implement a drone suitable for other tasks.
\end{abstract}

\section{INTRODUCTION}

Aerial survey mapping has traditionally been the most important application of photogrammetry. First aerial images were taken from a hot air balloon already in 1858 , but during the first world war, the trend of systematic aerial survey became stronger (Cohen, 2000). Modern full-scale aerial photogrammetry is often conducted with a fixed wing aircraft and a specialized camera equipment. Typical Ground Sample Distance (GSD) in urban applications is $10 \mathrm{~cm}$ (Meixner and Leberl, 2010). For example, with DMC III camera this is achieved with $2359 \mathrm{~m}$ flying altitude. In lower resolution campaigns, the flying altitude can be several kilometres making data acquisition efficient. Unfortunately, such equipments are expensive and highly regulated.

In 2006, Federal Aviation Administration issued certificates for military drones to be used for civilian purposes (Rao et al., 2016). In 2010s, commercial ready-to-fly Unmanned Aerial Vehicles (UAVs), such as Parrot AR.Drone (Bristeau et al. 2011), suitable for mapping purposes started to appear to market. These aircrafts can operate in lower altitudes in order to obtain the same or much smaller GSD (sub-centimeter) using normal off-the-shelf camera equipment resulting in much lower costs of operation. However, UAV survey typically covers much smaller area than a full-scale aerial survey. Like full-scale aircrafts, UAVs can be divided into fixed wing and rotary wing categories ( $\mathrm{Yu}$ and $\mathrm{Zhang}$, 2015). Fixed wing vehicles resemble airplanes while rotary wings are for example helicopters and multirotor aircrafts. Furthermore, a hybrid implementation consisting of both rotary wing and fixed wing applications is possible. This paper focuses on rotary wing vehicles.

UAVs have been utilized for many surveying applications such as mapping (e.g. Lin, 2008; Nex and Remondino, 2014), landslide monitoring (e.g. Walter et al., 2009), building inspection (e.g., Martinez-de Dios, and Ollero, 2006), postdisaster assessment (e.g. Bendea et al. 2008), monitoring safety hazards in construction sites (e.g. de Melo et al., 2017), monitoring crop conditions (e.g. Gago et al, 2015), and city and building modeling,(e.g. Wang and Li, 2007) just to mention few.

There is a wide range of commercially available UAVs designed for photogrammetric survey tasks with costs typically ranging from $1000 €$ to $30 \mathrm{k} €$. However, we feel that commercial UAVs do not offer best cost-quality ratio. UAV kits provide low-cost alternatives to build your own device, however, we believe that it is advantageous to be able to modify design according to specific needs.

The aim of this paper is to illustrate a workflow of how to design and implement a survey drone in the price range of couple of thousand euros while being able to meet the quality requirements of a much higher cost drone system. Basic design aspects of drone design are explained in practical manner, allowing the reader to modify the design to suit a specific type of drone application and finally a recommendation of a typical survey drone design is given. Furthermore, the design guidelines proposed in this article can be modified in order to tackle some specialized requirements for which there are no commercially available products in the market. For an example the open source approach used can facilitate designing a hybrid vehicle capable of both ground and air operation. However, the main focus in this paper is the survey drone application.

The main tools used in this paper include eCalch RC calculator and ArduPilot open source autopilot project. eCalc is used to simulate the operational limits of the drone using the xcopterCalc - Multicopter Calculator while ArduPilot is used to implement the autopilot and control functionality of the drone.

\section{DESIGNING A SURVEY DRONE}

The principle design question when starting the drone design is, what is the application? For an example, a recreational drone has different requirements than a delivery drone. 
Delivery drone should be able to carry as big payload for a given delivery route as possible while using the least amount of energy possible and maintaining overall cost efficiency. Recreational drone on the other hand should be fun to pilot. Our focus is on survey drones, which sets boundaries for design. Survey drone requirements are similar to the delivery drone in that they should be able to carry a significant payload in the form of survey equipment but the task is not to be able to carry as much as possible but rather carry what is needed for the longest time possible. This means the survey drone should be able to maintain an efficient operation within typical survey mission pace. In following, we describe our design process.

The first step is to define typical operational parameters. For the sake of simplicity, we will use nadir imaging in this example. The goal is to reach $2 \mathrm{~cm}$ GSD while staying well under $150 \mathrm{~m}$ flying altitude and travelling in such a slow pace that the motion blur is minimized in non-optimal lighting conditions. The goal of the optimization is such that the efficiency and thus the operational flight time is the longest for the optimized case but also relatively good for cases that are similar to the optimized case.

The most important factor to achieve the longest possible operational flight time is the proper combination of a motor and a propeller. Modern drones use brushless motors which are capable of over $90 \%$ efficiencies provided they are optimally operated. If a motor has too much or too little load, it will not achieve the best possible efficiency. The rule of thumb for the prop selection is the bigger the better. A long propeller will produce a big radius propeller disk, meaning a large area where lift is generated. This results in a big load of the motor which reduces the revolutions per minute of the motor. Thus, the choice of the motor(s) depends on the propeller used and vice versa. Table 1 illustrates the characteristics of a Multistar Elite 5008330 motor operating under optimal conditions and Table 2 shows how the characteristics change when the motor is fitted with a 18 " 5.5" pitch propeller. Even though the optimal efficiency of the motor is almost $90 \%$, it can be hard to reach that in practice and the component selection is always a compromise.

$\begin{array}{lc}\text { Motor @ Optimum Efficiency } \\ \text { Current: } & 8.58 \mathrm{~A} \\ \text { Voltage: } & 21.95 \mathrm{~V} \\ \text { Revolutions*: } & 6796 \mathrm{rpm} \\ \text { electric Power: } & 188.3 \mathrm{~W} \\ \text { mech. Power: } & 165.9 \mathrm{~W} \\ \text { Efficiency: } & 88.1 \%\end{array}$

Table 1. Optimal characteristics of a Multistar Elite 5008-330 motor using a $6 \mathrm{~S}$ battery pack.

$\begin{array}{lc}\text { Motor @ Hover } & \\ \text { Current: } & 2.94 \mathrm{~A} \\ \text { Voltage: } & 22.11 \mathrm{~V} \\ \text { Revolutions*: } & 2485 \mathrm{rpm} \\ \text { Throttle (log): } & 22 \% \\ \text { Throttle (linear): } & 39 \% \\ \text { electric Power: } & 65.0 \mathrm{~W} \\ \text { mech. Power: } & 53.2 \mathrm{~W} \\ \text { Power-Weight: } & 96.3 \mathrm{~W} / \mathrm{kg} \\ & 43.7 \mathrm{~W} / \mathrm{b} \\ \text { Efficiency: } & 81.8 \%\end{array}$

Table 2. Characteristics of a Multistar Elite 5008-330 motor using $18 " 5.5$ " pitch propeller and a $6 \mathrm{~S}$ battery pack.
In the first stage of the design, one should decide the target payload of the drone. In this example we are going to use a Sony Alpha 7R mirrorless full-frame camera and Zeiss Loxia 2.8/21 lens. The camera weights $465 \mathrm{~g}$ and the lens $394 \mathrm{~g}$. In addition, we use a 3 -axis gimbal which weights $300 \mathrm{~g}$, so the total payload is $1159 \mathrm{~g}$. The camera and gimbal can be seen in Fig 1.

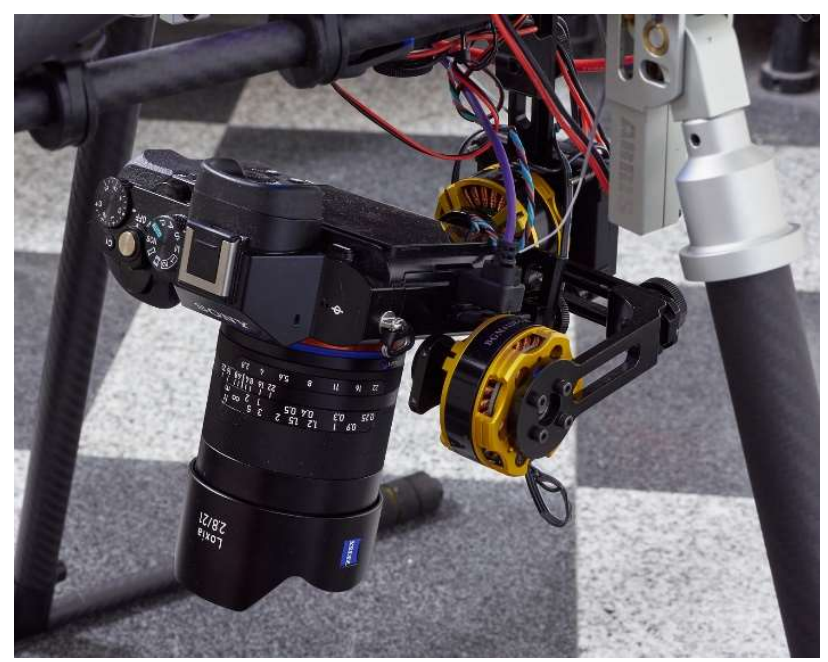

Figure 1. The 3-axis gimbal and the Sony Alpha 7R camera system with the Zeiss Loxia 2.8/21 lens.

This means that in addition to the lift needed to hover the drone and manoeuvre it, we need at least additional $1159 \mathrm{~g}$ lift to operate our survey equipment. Additionally, we should consider a safety margin. If one motor of a quadcopter malfunctions, the vehicle loses $1 / 4$ of the total thrust. This might lead into a situation where the lift is not sufficient to hover the drone anymore leading to an emergency landing or crash. Furthermore, a quadcopter operating with only three motors can be hard to control. It is possible to use an arbitrary number of motors but for each propeller blade, the efficiency is slightly compromised. Furthermore, while specialized vehicles such as tricopters exist, it is advised to use pairwise amount of motors in order to keep the drone balanced. As such, the drone design is always a compromise and should be optimized case by case. We utilized xcopterCalc simulation software for searching the optimal setup of our case.

In our example, we chose to use a hexacopter frame. Using six motors is less efficient than utilizing four motors, but it provides better safety in a motor malfunction case and allows a smaller drone footprint because a bigger percentage of the drone footprint can utilize propellers as the propeller blades are closer to each other. Once the frame type is decided, it is possible to simulate various options in xcopterCalc. We chose to further specify the exact frame model first and lock the selection. After comparing the specifications of various frames, we decided to use Tarot T960 frame. T960 has a wheelbase of $960 \mathrm{~mm}$ and thus allows to use up to 18 -inch propellers. In addition, most of the frame is constructed of carbon fiber so the durability of the frame should be good, and it is relatively lightweight. Rest of the components were chosen according to what resulted in the best simulated flight time overall.

We used xcopterCalc to simulate the flight time and the payload capabilities of our survey drone build before we had components of the build. We applied the specifications of component manufacturers to simulate the vehicle. Furthermore, we included a comparison to a quadcopter version of the drone. Finally, we 
included a verification of the simulation using real total weight once the drone was built. The drone is illustrated in Fig. 2.

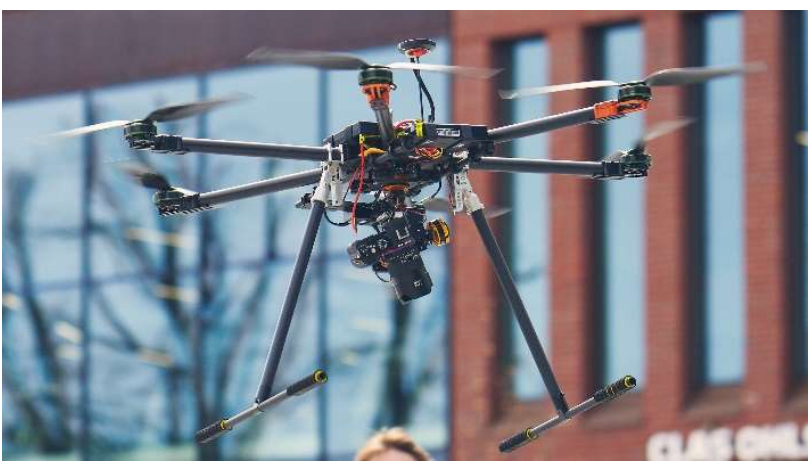

Figure 2. The finished survey drone in flight demonstration. (C) Aalto University

\subsection{Hexacopter drone simulation}

The input for the xcopterCalc is divided into five categories: General, Battery Cell, Controller, Motor and Propeller. After all the relevant information is filled, the program will simulate the design of the drone build. In the case there are problems, the program will give various alerts.

Starting from the general category we input $1050 \mathrm{~g}$ as the model weight without drive. This means that the battery, speed controllers and motors will be added to the $1050 \mathrm{~g}$ value to get the final weight of the vehicle. In this example, the number of rotors is six and frame size is $960 \mathrm{~mm}$ measured from the centres of two opposite side motor mounts. For illustrational purposes, we chose to use FCU (Flight Control Unit) tilt limit of $45^{\circ}$ to give more meaningful flight time graphs. Tilt limit can be a good idea also to minimize the risk of drone tipping over due to wind conditions or unstable payload configurations. We used field elevation of $50 \mathrm{~m}$ and air temperature of $20^{\circ} \mathrm{C}$ to simulate our typical operating conditions.

For the battery cell category, we chose the preset "LiPo $3700 \mathrm{mAh}-65 / 100 \mathrm{C}$ " and edited certain data fields to better represent the Turnigy nano-tech Ultimate 7500mah 2S2P 90C Hardcase Lipo Pack we are using. Batteries have cells connected in series and/or in parallel. In our case, each battery pack has two $3750 \mathrm{mAh}$ cells connected in series and two of them connected in parallel. The result is called $7500 \mathrm{mAh} 2 \mathrm{~S} 2 \mathrm{P}$ battery back and because of the parallel (2P) connection, the capacity is doubled. Similarly, the voltage of the pack is double of one cell because of the series connection (2S). In addition, the cell weight for our battery pack was lower than the default so that was adjusted to $83 \mathrm{~g}$. Now we could choose $6 \mathrm{~S} 2 \mathrm{P}$ or $6 \mathrm{~S} 4 \mathrm{P}$ configurations to reflect the actual battery configurations we had for a half (6S2P) and full (6S4P) battery configurations. Our chosen battery pack also has lower internal resistance than the default $0.003 \Omega$ but that is not relevant for this application.

Controller category consists of information about the speed controllers which operate the motors. In order to choose a right grade controller, one needs to know how much current the motors will draw. Oversizing a speed controller is possible and there is no other drawback than the increased cost and weight. We decided to use $30 \mathrm{~A}$ controllers and later in the simulation found that this was enough for this build. One should make sure that the chosen controller supports the operating voltage of the vehicle. This is not listed in xcopterCalc since it does not change the operating parameters of the drone.
The motor category is perhaps the most important one and also the most difficult to optimize. Manufacturer and type (Kv) dropboxes contain comprehensive selection of commercially available motors. In order to find an optimal motor, one needs to consider the size class of the motor. Different manufacturers can use slightly different systems but usually the first two digits of a motor mean the motor or rotor diameter and the following two the motor or rotor height. In our example after simulating many different configurations, we ended up with Multistar Elite 5008330 motor (Fig. 3). The motor diameter is $57 \mathrm{~mm}$ so it does not quite match the $50 \mathrm{~mm}$ specification so we can assume the rotor is $50 \mathrm{~mm}$. Similarly, the can height is $27 \mathrm{~mm}$ so $8 \mathrm{~mm}$ must be the rotor height. Usually frame manufacturers state recommended motors in the frame specification so that can be a good starting point in motor selection. The next parameter to consider is the Kv-rating of the motor. This indicates how many revolutions per minute will the motor reach in an unloaded case when given a certain voltage. For an example, the $\mathrm{Kv}$-rating of our motors is 330 and we use a nominal operating voltage of $22.2 \mathrm{~V}$ so the unloaded motors would spin at around 7300rpm. Generally big propellers require a low $\mathrm{Kv}$-rating while smaller propellers can utilize higher Kv-ratings and thus higher rpms. In our case, the 330 rating is on the high side of what is recommended for 18 " propellers we are using. Other motor parameters are not discussed in the scope of this paper. We recommend experimenting with selecting the motor from the dropbox and simulating how the motor fares in the simulation. Fig. 3 illustrates a Multistar Elite 5008-330 motor with a 18 " 5.5" pitch propeller mounted to the Tarot T960 hexacopter frame.

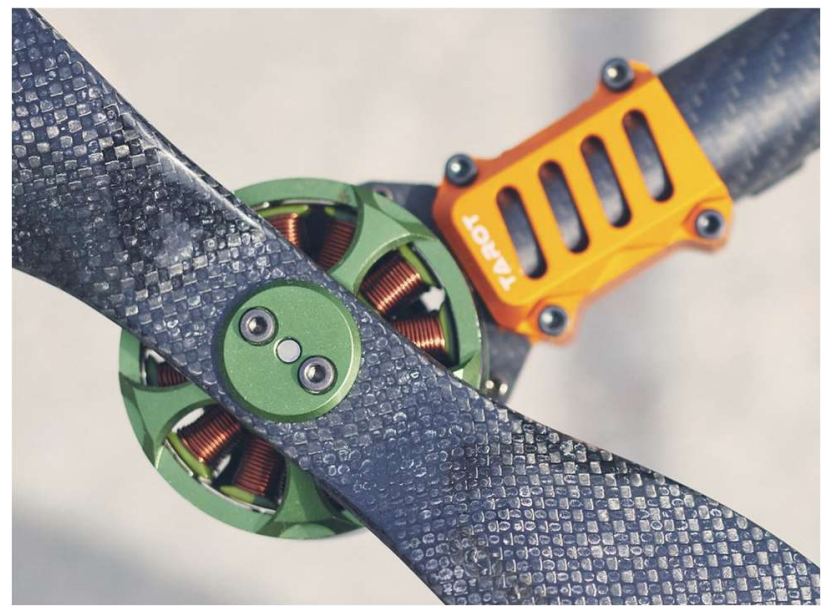

Figure 3. Multistar Elite 5008-330 fitted with a 18" 5.5" pitch propeller and attached to a Tarot T960 hexacopter frame. (C)

Aalto University

Propeller selection is highly dependent on the motor selection. If a propeller is changed, it is usually recommended to check if motors needs optimizing as well. The main propeller parameters are length in inches and pitch in inches. Length is the diameter of the disc that the propeller tip draws when being spinned. Pitch on the other hand is the distance the propeller would travel in an unrestricted case when it was turned one revolution. In practice, this means that higher pitch number have a steeper blade angle and thus require less rpm in order to generate lift. However, this also means that high pitch propellers stress the motors more than low pitch propellers. Propellers are easy to change after the drone is finished so it is also possible to design the drone for a set of propeller selections. 
Once all the relevant fields are filled, software will simulate the drone characteristics. At this point, if there are any errors or if the drone is about to operate outside the specification, the program will alert the user. In our case, the electric power at full throttle is near the limit so it is necessary to limit the max throttle in software later. Fig. 4 shows the xcopterCalc interface.

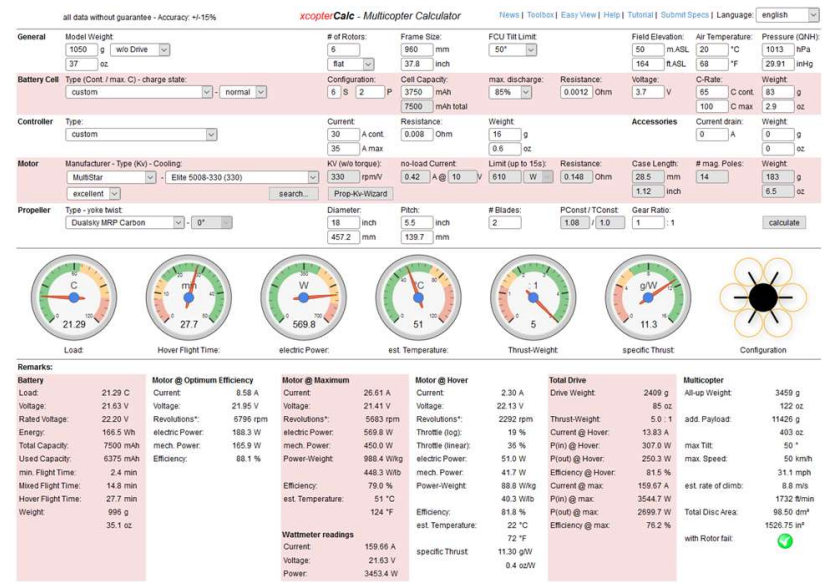

Figure 4. Simulation in xcopterCalc software.

\section{BUILDING A SURVEY DRONE}

The first stage of building the drone involves ordering the parts specified in the design stage and assembling them. This involves basic soldering skills and understanding of simple DC-circuits. In most cases, the frame kits or other parts do not include the connecting parts such as cabling. For the main power cables, one needs to take into account that the wires can withstand the current of the motors in full throttle case. There are charts available for quick reference and for more precise selection, it is possible to calculate the required wire gauge (Thue, 1998). In our case, we opted for 16AWG wire for most parts of the drone and a thicker 10AWG wire between the battery and the power distribution board. 26AWG wires were used for signal cabling. Initial wiring layout is visible in Fig 5.

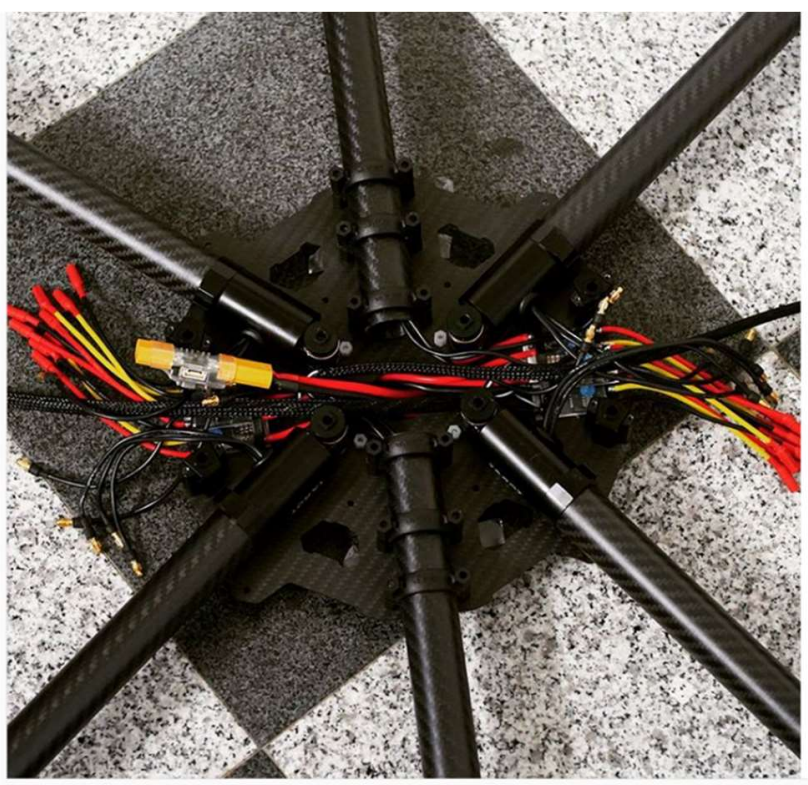

Figure 5. The initial wiring layout of the drone without the bottom plate of the frame.
While it is not strictly required, it is a good practice to connect the battery to the speed controllers and flight controller via a power distribution board. We utilized a universal 12-way 120A power distribution hub (product SKU: 206000016-0). This board includes two Battery Elimination Circuits (BEC), which provide fixed $5 \mathrm{~V} 3 \mathrm{~A}$ and $10 \sim 20 \mathrm{~V}$ adjustable (36W max.) outputs for auxiliary devices. We used the $5 \mathrm{~V}$ output to power the flight controller and the adjustable output for the camera gimbal. The power distribution board is illustrated in Fig. 6.

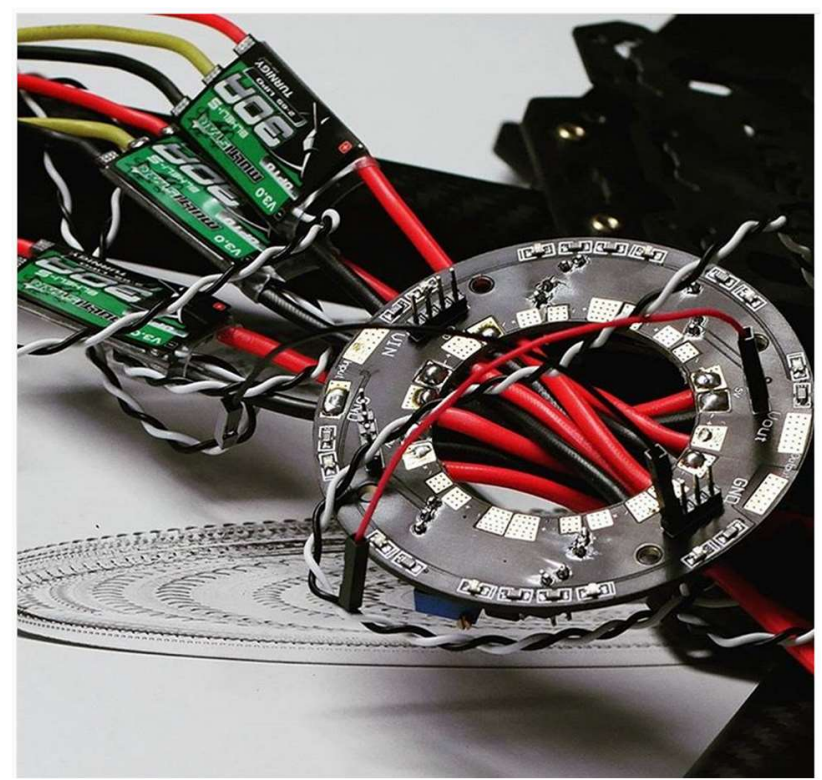

Figure 6. Power distribution board and three 30A speed controllers.

Flight controller unit is an important part of a survey drone. It controls all the functionality of the drone and most of the auxiliary devices are connected to the FCU. There are many different FCUs available in the market and one should choose the appropriate one depending on the application (Colomina, 2014). We chose to use Pixhawk 2 FCU, which was just released when we were about to order the parts for our build. Pixhawk 2 is a successor for Pixhawk which in turn is based on ArduPilot open source platform. Pixhawk 2 uses Triple Redundant IMU system with 3 accelerometers, 3 gyroscopes, 3 magnetometers and 2 barometers. In a case of a sensor failure, it is possible to disable the faulty sensor and continue with an emergency landing without loosing control of the drone. This is an important safety feature especially when flying over crowds with a special permit. Furthermore, we had prior experience in previous ArduPilot FCU.

For a survey drone, a GPS receiver is often used. Some FCUs bundle the GPS antenna as a kit as was the case with the Pixhawk 2 bundle we used. The antenna we chose also houses a compass which is used to control the heading of the drone in conjunction with the gyros. Real-Time Kinematic (RTK) GPS is also possible and makes direct georeferencing of the survey mission feasible. If the camera is also controlled by the FCU, it is possible to geotag the images using the on-board GPS data. Fig. 7 illustrates the GPS and $433 \mathrm{MHz}$ antennas mounted next the the Pixhawk 2 FCU.

In addition, the FCU can control a number of auxiliary devices. This is usually done utilizing a servo signal. For an example, the camera gimbal controller we apply takes three servo signals as inputs to control the three rotations. This makes it possible to 
automatically aim the camera towards a region of interest and keep track of which direction the camera is pointing. In our build, the FCU also triggers the camera with a servo signal. In order to transform the servo signal into a camera trigger signal, a Seagull \#MAP2 camera trigger device (Fig. 8) is used.

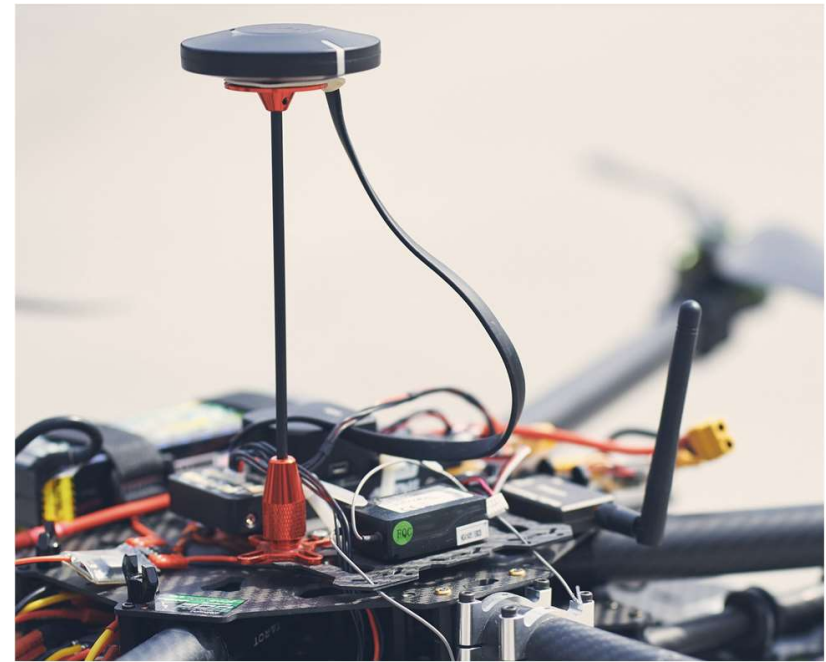

Figure 7. GPS (left) and $433 \mathrm{MHz}$ (right) antennas. (C) Aalto University

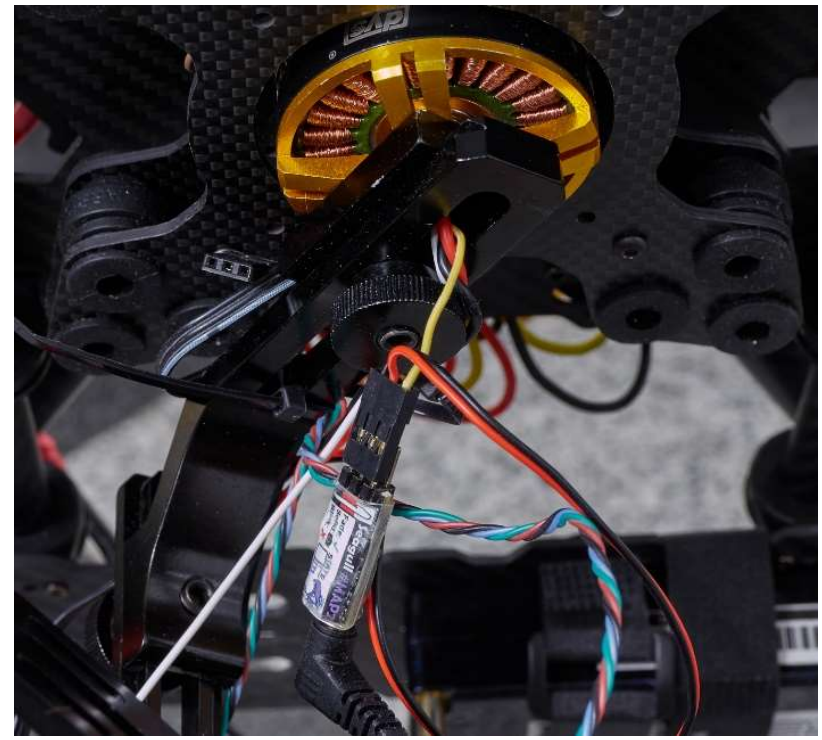

Figure 8. 3-axis Brushless gimbal yaw-motor and Seagull \#MAP2 camera trigger.

For a remote control of the drone, at least one transmitter/receiver pair is needed. The basic functionality is offered by connecting a RC-receiver to the FCU and using RC-transmitter to connect to the receiver. There is a wide selection of different $\mathrm{RC}$ radio systems available and the detailed discussion about them is not in the scope of this article. We chose to use the DEVO10 transmitter and the RX703A receiver. We already had the DEVO10 from a previous project and the RX703A was chosen because it supports a data bus connection, which is supported by Pixhawk 2, making it possible to use only one cable to connect the receiver to the FCU. In addition, we connected $433 \mathrm{MHz}$ telemetry radio, which acts as a transmission system between the FCU and a Ground Control Station (GCS). The assembled drone is illustrated in Fig. 9. The battery layout is for the $6 \mathrm{~S} 2 \mathrm{P}$ configuration and asymmetrical in purpose. The battery packs were used to place the point of balance in the centre of the drone resulting in more efficient and safer operation. The initial imbalance of the drone was caused by the survey equipment.

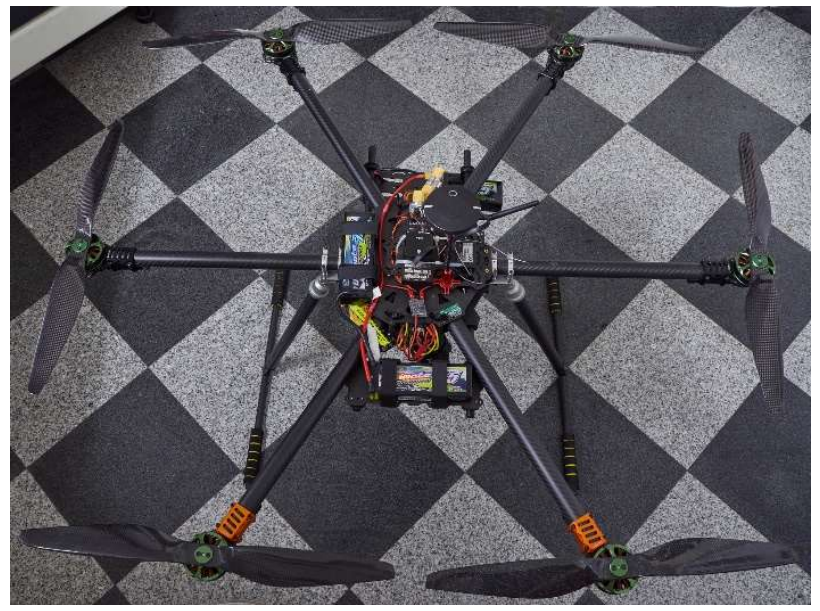

Figure 9. The finished survey drone.

\section{RESULTS}

As a result of this paper, we briefly represent validation of the simulated flight time in a real survey mission using a cross grid flight plan and a 3-axis gimbal stabilised Sony Alpha 7R camera system with Zeiss Loxia 2.8/21 lens as a payload. The drone was fitted with a $6 \mathrm{~S} 2 \mathrm{P}$ battery pack. Fig. 10 shows the simulated expected flight times for the survey drone. The real maximum flight time is expected to be between the no drag and standard drag cases.

The survey site is the Träskända manor in Espoo, Finland. The survey was conducted as a cross grid oblique imaging survey flight and the data was later processed in Agisoft Metashape to form a 3D point cloud. The total length of the flight path was $2771 \mathrm{~m}$. Fig. 10 shows the flight plan waypoints in green and the designed flight path in yellow. The arrows indicate the direction of travel. The realised flight path is shown in magenta. The mission cruise speed was set to $5 \mathrm{~m} / \mathrm{s}$ and apart from the manual take-off and landing, ArduPilot autopilot controlled the drone. A waypoint marked $\mathrm{H}$ indicates the planned take-off and landing position but that was changed to near the waypoint 69 as can be seen from the magenta drone trajectory path.

The expected flight time according to the simulation was between 9 and 15 minutes according to the xcopterCalc Range Estimator graph presented in Fig. 10. The survey mission took 13 minutes from take-off to landing which illustrates that the drone was able to conduct a survey mission as planned utilising the simulated flight time results. Wind conditions were mild during the whole mission. After the survey mission, the battery pack voltage was measured at $22.22 \mathrm{~V}$ which indicates approximately $15 \%$ battery charge was left.

According to the simulation the mixed flight time without payload using 6S2P (half) battery is $14.8 \mathrm{~min}$ and hover flight time is $27.7 \mathrm{~min}$. In practice, the flight time when flying a survey mission can be expected to be between these two values. If the camera and gimbal are added, the mixed flight time drops to $11.5 \mathrm{~min}$ and hover flight time to $17.9 \mathrm{~min}$ respectively. 6S4P (full) battery would result in $18.6 \mathrm{~min}$ mixed and $25.8 \mathrm{~min}$ hover flight times with payload and 23.3min mixed and $36.6 \mathrm{~min}$ hover flight times without payload. 


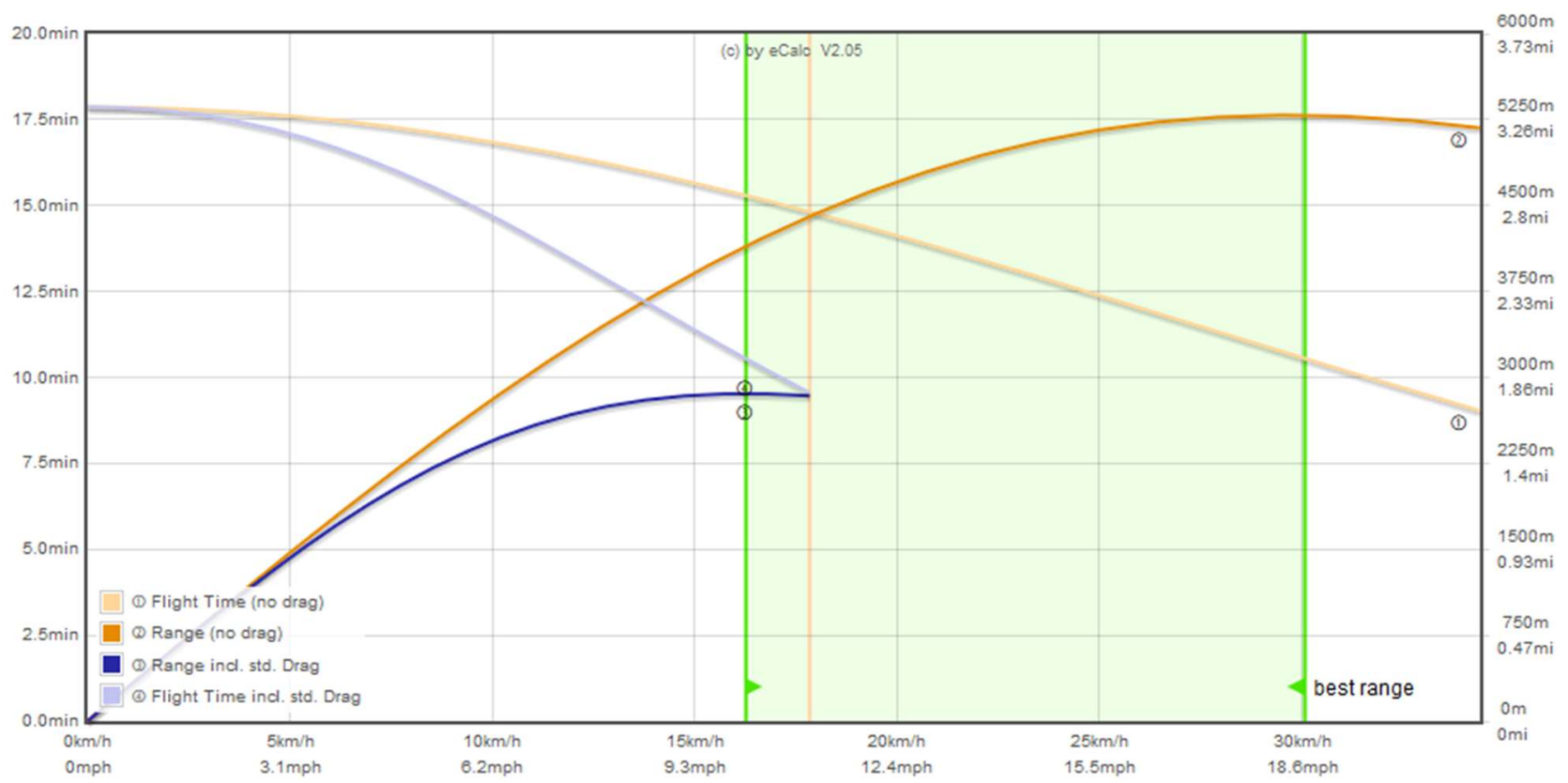

Figure 10. Simulated flight time and distance graph for the survey hexacopter build.

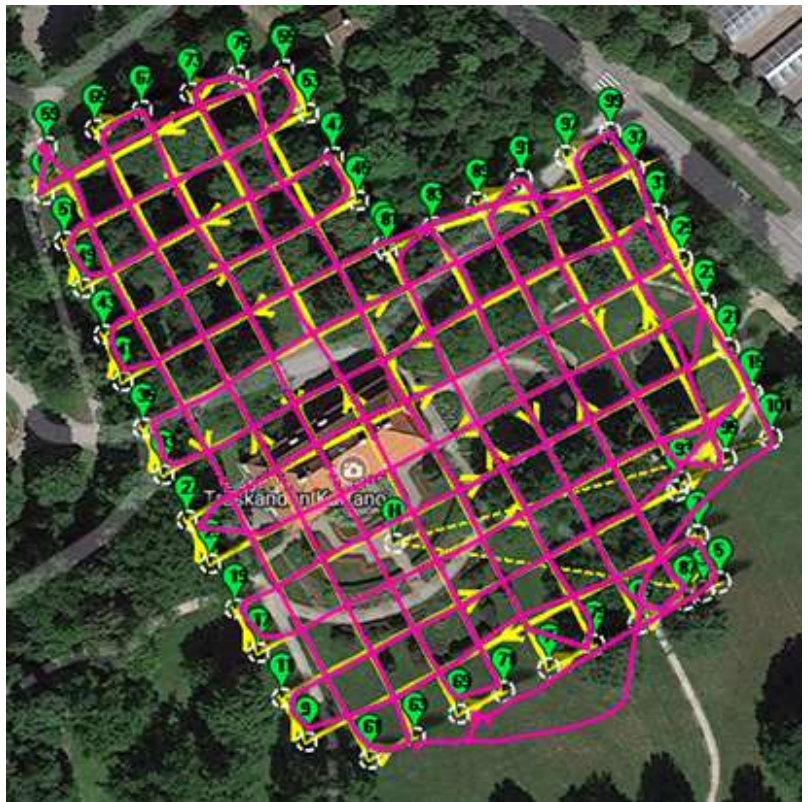

Figure 11. Flight plan and realised flight path of an example survey mission in Träskända. Planned flight path is shown in yellow and the drone flight path is illustrated in magenta.

We have not verified the flight times with a 6S4P battery pack in practice. Hover flight time indicates the time the drone can stay airborne in a static position while mixed flight time illustrates dynamic flight. However, for a survey application a stable relatively slow cruise speed is desired and thus the range estimator graph is highly useful.

Another important result for a survey drone is the payload capability of the designed vehicle. In the case of our hexacopter build, the drone can support a payload of up to $11 \mathrm{~kg}$. However, that would require full throttle and cripple the flight time to less than 3 minutes.

\subsection{Quadcopter simulation comparison}

As a comparison we present a simulation of what happens when the number of motors of our hexacopter build is changed to four, corresponding to the quadcopter configuration. As a result of fewer motors, the total maximum lift of the drone is reduced from $14.9 \mathrm{~kg}$ to $10.0 \mathrm{~kg}$ and the motors need to spin faster to reach the desired lift. This changes the operating point of the motors so that the efficiency in hover is reduced from $81.6 \%$ to $80.9 \%$. Drive weight is reduced from $2409 \mathrm{~g}$ to $1971 \mathrm{~g}$. Flight time at $5 \mathrm{~m} / \mathrm{s}$ cruise speed is changed from between 9 and 15 minutes to 10 and 14 minutes. However, the flight time differences are within the xcopterCalc specified error tolerances. The major difference between the builds is the payload headroom and safety. In a case of a motor failure, the quadcopter build is likely to crash while hexacopter should be able to resist losing control. We found the simulation provided a practical way to find out whether a certain scenario is feasible or not by altering the design and examining the simulation results.

\section{DISCUSSION}

In practice, the manufacturer specified weights might not be accurate so once the drone is built, it is recommended to correct the all-up weight with real values to xcopterCalc before the first flight to make sure the design weight matches with the actual weight of the vehicle. In our case, the finished drone is heavier than the design at $6 \mathrm{~kg}$ all-up weight, translating to simulated $8.8 \mathrm{~min}$ mixed and $12.0 \mathrm{~min}$ hover flight times with $6 \mathrm{~S} 2 \mathrm{P}$ battery. This is caused by the design weight not including cabling, remote operated landing gear, wiring, GPS antenna, radio equipment and other auxiliary equipment. Thus, we recommend always weighing the real all-up weight of the vehicle and considering the safety limits using simulation before the actual survey flight. As a default eCalc flight times assume that $85 \%$ of the battery capacity is used during the flight. This indicates that it is possible to exceed the eCalc specified flight times, but it is not advised because LiPo batteries have a reduced lifespan if they are regularly fully drained. In addition, eCalc states that all the simulations are within a $+/-15 \%$ accuracy. It is recommended to 
conduct a series of test flights to verify the simulation results. When surveying larger areas, we plan to use at least 6S4P battery pack which should extend the flight time near 20 minutes mark.

In our case, the propeller selection was limited by the availability of 18 " propellers and we were forced to choose higher pitch propellers than what would have been optimal for our application. Changing the propellers is straightforward and we plan to experiment with different propeller configurations later.

The distribution board we chose can handle currents up to $120 \mathrm{~A}$ and according to xcopterCalc our drone draws up to $160 \mathrm{~A}$ at max throttle. Thus, it is advised to limit the maximum throttle in software. However, even if the distribution board is rated for $120 \mathrm{~A}$, it can most likely sustain significantly more than that for brief amounts of time. Table 3 illustrates the simulated motor characteristics in a full throttle case. Wattmeter readings indicate the total electric power during a full throttle scenario while Motor (a) Maximum shows the characteristics of one motor.

$\begin{array}{lc}\text { Motor @ Maximum } & \\ \text { Current: } & 26.61 \mathrm{~A} \\ \text { Voltage: } & 21.41 \mathrm{~V} \\ \text { Revolutions*: } & 5683 \mathrm{rpm} \\ \text { electric Power: } & 569.8 \mathrm{~W} \\ \text { mech. Power: } & 450.0 \mathrm{~W} \\ \text { Power-Weight: } & 840.8 \mathrm{~W} / \mathrm{kg} \\ & 381.4 \mathrm{~W} / \mathrm{b} \\ \text { Efficiency: } & 79.0 \% \\ \text { est. Temperature: } & 51^{\circ} \mathrm{C} \\ & 124^{\circ} \mathrm{F} \\ & \\ \text { Wattmeter readings } & \\ \text { Current: } & 159.66 \mathrm{~A} \\ \text { Voltage: } & 21.63 \mathrm{~V} \\ \text { Power: } & 3453.4 \mathrm{~W}\end{array}$

Table 3. Multistar Elite 5008-330 motor characteristics at full throttle using 18 " 5.5 " pitch propeller. Wattmeter readings are for all six motors combined.

There are example builds for different FCUs to act as a starting point to determine the drone stabilisation parameters and other settings. Care should be taken when using these if there are significant design differences between the example and the actual drone build. For an example, a survey drone is likely to have a higher trust to weight ratio without payload than a recreational drone. This results in more pronounced movements with a certain throttle input. FCU controls the drone stabilisation automation and needs to know how much throttle input is needed to correct a certain degree of swing to some direction. If too much correction is applied, the drone will overcorrect the movement and end up oscillating. This is because the FCU sensors can only detect the drone pose after the correction happened. Thus, it is important to calibrate the stabilisation gains of the FCU before operating the drone in survey missions. In the ArduPilot platform, there is an automatic calibration sequence to tackle with this issue, but the initial stabilisation gains need to be set before that in order to get the drone airborne to run the calibration.

In a typical survey mission, the flight plan is made beforehand and uploaded to the drone FCU using a cable or $433 \mathrm{MHz}$ telemetry connection. During the flight, a cable connection is unfeasible but $433 \mathrm{MHz}$ connection can be used to modify the flight plan or operate the drone using computer in case the main transmitter fails. $433 \mathrm{MHz}$ connection is also used to provide the operator with real-time statistics about the drone such as the battery voltage, altitude, drone pose and such. We feel this is an important safety aspect and strongly recommend including a telemetry connection for any survey drone build.

\section{CONCLUSIONS}

The paper presents a workflow of designing and simulating a survey drone design. The designed vehicle is constructed and tested in real world survey mission to verify the simulation results. The drone was able to successfully conduct the survey mission within the simulation result specifications. We conclude that using eCalc as a tool to design a survey drone works in practice and makes the component selection much more straightforward. The flight time and payload capabilities of the drone matched the simulated results within the accuracy stated by the eCalc specification. Simulation provides a useful tool to see how the drone works under certain conditions such as working in extreme temperatures or high elevation locations as well as under heavy payloads. Even though our example was a survey drone, it is possible to use the same principles to design and implement a drone suitable for other tasks.

\section{ACKNOWLEDGEMENTS}

This research was funded by the Centre of Excellence in Laser Scanning Research (CoE-LaSR) (No. 272195), Strategic Research Council project "Competence-Based Growth Through Integrated Disruptive Technologies of 3D Digitalization, Robotics, Geospatial Information and Image Processing/ Computing-Point Cloud Ecosystem", pointcloud.fi (No. 293389). The support from the European Regional Development Fund (ERFD) for projects 3D Culture Hub (A72980) is acknowledged.

\section{REFERENCES}

Bendea, H., Boccardo, P., Dequal, S., Giulio Tonolo, F., Marenchino, D., Piras, M., 2008. Low cost UAV for post-disaster assessment. The International Archives of the Photogrammetry, Remote Sensing and Spatial Information Sciences. Vol. XXXVII. Part B8. Beijing, pp. 1373-1379.

Bristeau, P.-J., Callou, F., Vissière, D., Petit, N., 2011. The Navigation and Control technology inside the AR.Drone micro UAV. Proceedings of the 18th World Congress, The International Federation of Automatic ControlMilano (Italy), pp. 1477-1484.

Cohen, C. J., 2000. Early history of remote sensing. In Proceedings of 29th Applied Imagery Pattern Recognition Workshop, October 16-18, 2000, pp. 3-9.

Colomina, I., Molina, P., 2014. Unmanned aerial systems for photogrammetry and remote sensing: A review. ISPRS Journal of photogrammetry and remote sensing, 92, pp.79-97.

Gago, J., Douthe, C., Coopman R. E, Gallego, P.P., Ribas-Carbo, M., Flexas, J., Escalona, J., Medrane, H., 2015. UAVs challenge to assess water stress for sustainable agriculture. Agricultural Water Management, 153, pp. 9-19. 
Martinez-de Dios, J.R., Ollero, A., 2006. Automatic detection of windows thermal heat losses in buildings using UAVs. Proc. IEEE Automation Congress, WAC'06, Budapest, Hungary, pp. $1-6$.

de Melo, R.R.S., Costa, D.B., Alvares, J.S., Irizarry, J., 2017. Applicability of unmanned aerial system (UAS) for safety inspection on construction sites. Safety Science, 98, pp. 174185 .

Lin, Z., 2008. UAV for mapping-low altitude photogrammetric survey, The International Archives of the Photogrammetry, Remote Sensing and Spatial Information Sciences. Vol. XXXVII. Part B1. Beijing, pp. 1183-1186.

Meixner, P., Leberl, F., 2010. Describing buildings by 3dimensional details found in aerial photography. Int. Arch. Photogramm., Remote Sens. Spatial Inf. Sci., XXXVIII (Part 3A), pp. 151-156.

Nex, F., Remondino, F., 2014. UAV for 3D mapping applications: a review. Applied Geomatics, 6, pp. 1-15.

Rao, B., Gopi, A., Maione, R., 2016. The Societal Impact of Commercial Drones. Technology in Society, 45, pp. 83-90.

Thue, W.A. (ed.) 1998, Electrical Power Cable Engineering. Second Edition, CRC Press, New York., 328 pages.

Walter, M., Niethammer, U., Rothmund, S., Joswig, M., 2009. Joint analysis of the Super-Sauze (French Alps) mudslide by nanoseismic monitoring and UAV-based remote sensing. First Break 27, pp. 53-60.

Wang, J., Li, C., 2007. Acquisition of UAV images and the application in 3D city modeling, Proc. SPIE 6623, International Symposium on Photoelectronic Detection and Imaging: Image Processing, 11 pages.

Yu, X., Zhang, Y., 2015. Sense and avoid technologies with applications to unmanned aircraft systems: Review and prospects. Progress in Aerospace Sciences, 74, pp. 152-166. 